\title{
Status of ESBL Producing Bacteria Isolated from Skin Wound at a Tertiary Care Hospital in Bangladesh
}

\author{
Taslima Yasmin'1, Md. Abdullah Yusuf', Mohammed Abdun Nur Sayam³, Rezwana Haque, \\ Golam Mowla ${ }^{5}$ \\ ${ }^{1}$ Department of Microbiology, North Bengal Medical College, Sirajgonj, Bangladesh \\ ${ }^{2}$ Department of Microbiology, National Institute of Neurosciences \& Hospital, Dhaka, Bangladesh \\ ${ }^{3}$ Narayngonj General Hospital, Narayngonj, Bangladesh \\ ${ }^{4}$ Department of Microbiology, Islami Bank Medical College, Rajshahi, Bangladesh \\ ${ }^{5}$ Department of Community Medicine, Mymensingh Medical College, Mymensingh, Bangladesh \\ Email: taslimashanta@yahoo.com,ayusuf75@yahoo.com,drsayam38@gmail.com, \\ sharifibs@gmail.com,gmowla37@yahoo.com
}

Received 1 October 2015; accepted 4 December 2015; published 7 December 2015

Copyright (C) 2015 by authors and Scientific Research Publishing Inc.

This work is licensed under the Creative Commons Attribution International License (CC BY).

http://creativecommons.org/licenses/by/4.0/

(c) (i) Open Access

\section{Abstract}

Background: ESBL producing bacteria are increasing with an alarming rate with a wide range of infections. Objective: The purpose of the present study was to see the status of ESBL producing bacteria isolated from skin wounds. Methodology: This cross sectional study was conducted in the Department of Microbiology at Mymensingh Medical College, Bangladesh from January 2011 to June 2011 for a period of 6 months. All the patients, at any age with both sexes presented with skin wound infection, were taken as study population. Wound swab was taken from all patients. Specimens were processed and bacteria were isolated and identified according to standard procedure. The ESBL status was confirmed by double disc diffusion test (DDDT) and minimum inhibitory concentration (MIC) by agar dilution method by standard procedure according to Clinical Laboratory Standard Institute (CLSI). Antimicrobial resistance was done by disc diffusion method. Result: A total number of 84 wound swabs were taken of which the most common ESBL producing bacteria were Esch. coli (61.5\%), Proteus species (78.3\%) and Klebsiella species (88.9\%). All the isolates were sensitive to imipenem and nitrofurantoin followed by amikacin (92.9\%). Conclusion: In conclusion, ESBL producing $E$. coli is the most common bacteria causing skin wound infection followed by Proteus species with a reduced sensitivity towards antibiotics.

\section{Keywords}

Extended Spectrum $\beta$-Lactamases, Escherichia coli, Klebsiella Species, Gram Negative Bacilli 


\section{Introduction}

Skin infections are very frequently encountered in clinical practice and are one of the most common sites of bacterial infections [1]. These infections are also among the most common indications for antibiotic therapy and hospital admissions [2]. However, increased use of antibiotics, particularly the third generation cephalosporin, has been associated with the emergence of $\beta$-lactamases [2]. These enzymes have serine at their active site and attack the amide bond in the beta lactam ring [3]. ESBLs are enzymes that mediate resistance to third generation cephalosporin as well as monobactams. Furthermore, these are inhibited in vitro by $\beta$-lactamase inhibitors such as clavulanic acid and tazobactam [4]-[6].

ESBLs have been reported worldwide in many different genera of Enterobactericeae and Pseudomonas species [7]-[9]. However, these are the most common in Klebsiella pneumoniae and Escherichia coli [10]-[12]. TEM-1 is the first plasmid mediated $\beta$-lactamase in Gram-negative bacteria [13]. Afterwards it was detected from Klebsiella species in Germany and France [5]. Later, ESBLs have been reported from all over the world. The true incidence is difficult to determine because of the difficulty and inconsistencies [13]. The high prevalence of ESBL genes indicates that the empirical treatment of serious infections and $\beta$-lactamase antibiotics except carbapenems is seriously compromised [14].

In Bangladesh Rahman et al. [15] reported 43.2\% and 39.5\% ESBL producing Esch. coli and K. pneumoniae respectively from urine, wound swab and pus. Skin wound is mainly caused by Gram Positive Cocci (GPC), but we take here only Gram Negative Bacilli (GNB) as ESBLs are mainly found in GNB. This is alarming to the patients as well as to the clinician. Proper burden of ESBL is needed to explore. Therefore, the present study was undertaken to see the status of ESBL producing bacteria isolated from skin wounds.

\section{Methodology}

This cross sectional study was conducted in the laboratory of the Department of Microbiology at Mymensingh Medical College, Mymensingh. This study was carried out from January 2011 to June 2011 for a period of 6 months. All the patients at any age with both sexes presented with skin wound infection who were attended at the OPD as well as the patients who were admitted in the IPD were taken as study population. Wound swab from all patients was taken by sterile swab stick. Thereafter, specimens were processed and bacteria were isolated and identified according to standard procedure [16]. Only GNB were taken in this study. All samples were routinely cultured on MacConkey's agar media and blood agar plates at $37^{\circ} \mathrm{C}$ aerobically for 18 hours. Gram negative isolates were further identified by standard biochemical tests [16]. The susceptibility test was determined by Kirby Bauer method on Muller Hinton agar medium [17]. Two diagnostic tests were performed for phenotypic detection of ESBL producing bacteria which were disc diffusion test (DDT) [18] used as screening test for ESBL production and double disc diffusion test (DDDT) [18] as confirmatory test. MIC reduction methods [19] were also performed for detection of ESBL according to CLSI17 for further confirmation for ESBL. Screened for ESBL production by using disc diffusion test on Muller-Hinton agar where isolates showing inhibition zone size of $\geq 22 \mathrm{~mm}$ with ceftazidime ( $30 \mu \mathrm{g}$ ), $\geq 25 \mathrm{~mm}$ with ceftriaxone ( $30 \mu \mathrm{g}), \geq 27 \mathrm{~mm}$ with cefotaxime ( $30 \mu \mathrm{g}), \geq 27 \mathrm{~mm}$ with Aztreonam $(30 \mu \mathrm{g})$ were suspected for ESBL production 18. ATCC 25,922 of E. coli was used as positive control strains. ATCC 25,922 of E. coli was used as a negative control. In double disk diffusion test (DDDT) a disc of ceftazidime $(30 \mu \mathrm{g})$, cefotaxime $(30 \mu \mathrm{g})$ alone and a disc of ceftazidime and cefotaxime in combination with clavulanic acid $(30 / 10 \mu \mathrm{g})$ were used for each isolates. Both the discs were placed $25 \mathrm{~mm}$ apart, centre to center, on a lawn culture of the test isolate on Muller Hinton agar plate and incubated overnight at $37^{\circ} \mathrm{C}$. A $\geq 5 \mathrm{~mm}$ increase in zone diameter for either antimicrobial agent tested in combination with clavulanic acid versus its zone when tested alone was designated as ESBL positive 18. In MIC reduction test break point of ceftazidime was $\geq 8 \mu \mathrm{g} / \mathrm{ml}$ was taken ESBL positive 19 . Statistical analysis was performed by SSPS 19.0. Qualitative variables were expressed by frequency and percentage.

\section{Results}

Among 84 specimens, wound swab were 45 (53.57\%) and pus was 39 (46.42\%). Majority bacterial isolates were ESBL positive which was 61 (72.6\%) cases. The most common isolated bacteria was Esch. coli which was 39 (46.4\%) followed by Proteus species, Klebsiella species, Pseudomonas species which were 23 (27.4\%), 9 (10.71\%) and 6 (7.1\%). Enterobacter species and Citrobacter species were also detected in 7 (8.3\%). ESBLs 
production rate was higher among Klebsiella species (88.9\%) followed by Proteus species (78.26\%), E. coli (61.53\%), Pseudomonas species (100\%) and others (71.42\%) (Table 1).

The antimicrobial susceptibility patterns of ESBL producers were measured. ESBL positive bacteria were $100.0 \%$ sensitive to imipenem and nitrofurantoin. On the other hand Aztreonam and Piperacillin were $100.0 \%$ resistant to ESBL producing bacteria. However, more than 80.0\% resistance was found from Ampicillin (89.5\%), Amoxiclav (89.3\%), Ceftazidime (81.2\%), Ceftriaxone (80.8\%), Ciprofloxacin (84.4\%) and Co-trimoxazole (85.1\%). Quinolone and aminoglycosides were more resistant among ESBLs producers than non ESBL producers (Table 2).

\section{Discussion}

Skin wound infection is very common [4]. Multiple bacteria cause this infection. The irrational use of antibiotics to this infection cause partial elimination of susceptible of bacteria and favours the survival and multiplication of drug resistant bacteria in most of the occasions [15]. Proper use of antibiotics is very important for various reasons.

Table 1. Detection rate of different isolates in the study population.

\begin{tabular}{cccc}
\hline Name of the organism & ESBL positive & ESBL negative & Total \\
\hline E. coli & $24(61.5 \%)$ & $15(38.5 \%)$ & $39(100.0 \%)$ \\
Proteus species & $18(78.3 \%)$ & $5(21.7 \%)$ & $23(100.0 \%)$ \\
Klebsiella species & $8(88.9 \%)$ & $1(11.1 \%)$ & $9(100.0 \%)$ \\
Pseudomonas species & $6(100.0 \%)$ & $0(0.0 \%)$ & $6(100.0 \%)$ \\
Others & $5(71.4 \%)$ & $2(28.6 \%)$ & $7(100.0 \%)$ \\
Total & $\mathbf{6 1}(\mathbf{7 2 . 6 \% )}$ & $\mathbf{2 3 ( 2 7 . 4 \% )}$ & $\mathbf{8 4}(\mathbf{1 0 0 . 0} \%)$ \\
\hline
\end{tabular}

${ }^{*}$ E. coli $=$ Escherichia coli.

Table 2. Antimicrobial resistance pattern of ESBLs producer and non ESBLs producer among skin wound isolates.

\begin{tabular}{ccc}
\hline Antibiotics tested & $\begin{array}{c}\text { Antimicrobials resistance to } \\
\text { ESBL positive }(\mathrm{n}=61)\end{array}$ & $\begin{array}{c}\text { Antimicrobials resistance to } \\
\text { ESBL negatives }(\mathrm{n}=23)\end{array}$ \\
\hline Ampicillin & $89.5 \%$ & $58.2 \%$ \\
Amoxiclave & $89.3 \%$ & $1.9 \%$ \\
Amikacin & $5.6 \%$ & $21.0 \%$ \\
Azithromicine & $48.1 \%$ & $100 . \%$ \\
Aztreonam & $100.0 \%$ & $50.8 \%$ \\
Ceftazidime & $81.2 \%$ & $31.8 \%$ \\
Ceftriaxone & $80.8 \%$ & $38.1 \%$ \\
Cefotaxime & $77.3 \%$ & $38.0 \%$ \\
Gentamicine & $44.7 \%$ & $74.4 \%$ \\
Ciprofloxacin & $84.4 \%$ & $0.0 \%$ \\
Nitrofurantoin & $0.0 \%$ & $100.0 \%$ \\
Piperacillin & $100.0 \%$ & $0.0 \%$ \\
Imipenem & $0.0 \%$ & $46.3 \%$ \\
Co-trimoxazole & $85.1 \%$ & \\
\hline
\end{tabular}


In the present study, the isolated gram negative bacteria from skin wound were E. coli (46.4\%), Klebsiella species (10.7\%), Proteus species (27.4\%), Pseudomonas species (7.1\%) and others (8.3\%) which correlate with the study done by Haque et al. [20] in the same hospital. Klebsiella species (88.9\%) was the leading ESBL producers from skin wound followed by Proteus species (78.3\%), Enterobacter species (71.4\%), E. coli (61.5\%) and Pseudomonas species (100.0\%). In another study in Bangladesh Haque and Salam21 have reported that ESBL production for Klebsiella species was 57.9\% followed by Proteus species (50.0\%), E. coli (47.8\%) and Pseudomonas species (31.3\%) [21]. The high frequency of ESBLs in Klebsiella species is of great concern since infections caused by this bacterium were very common. In addition to that resistance of the organism may be due to the presence of some virulence factor like hyper viscosity, polysaccharide capsule and production of endotoxin, carbapenemase, which make it more resistant [7]. Furthermore, they also spread easily with pathogenic and efficient at acquiring and disseminating resistance plasmid [3] [13].

In this present study the occurrence of ESBLs observed among the Pseudomonas species may not reflect the actual picture because of very small sample size. Prevalence of ESBL in Bangladesh was 41.1\% [14] and 41.7\% [21] in 2004 and 2010 respectively. In few studies from Pakistan [22] it was found 40.0\% and two other studies were 43\% [23] and 58.7\% [24] ESBLs producers. Several studies from India reported as ESBLs producers were 40.8\% [10], 51.4\% [25] and 53.8\% [26] respectively. In Nigeria [13] ESBL production rate was 66.7\%. The frequency of ESBL producer in the present study was $72.0 \%$ in general which was higher than the previous studies in Bangladesh [21], India [26] and other countries [22]. It may be due to steadily increasing the incidence of ESBL producing strains among the clinical isolates. Another two studies in Iran [27] and India [28] were reported $96.0 \%$ and $97.0 \%$ respectively. In the present study, some samples were taken which were sensitive to 3GCs and subsequently showed positive for ESBLs production by DDDT (40.0\%) [29], as because failure to detect ESBL production by routine disc-diffusion tests has been well documented [30] [31]. However, the study that has been reported in Iran [27] and India [28] showed higher rates of ESBL producing bacteria than the present study and it may be due to the fact that they consider only 3GCs resistant organisms. Occurrence and distribution of ESBLs differs from country to country and from hospital to hospital [24].

Development of multidrug resistance in clinical isolates like Pseudomonas species and Klebsiella species has been reported in Bangladesh [32] ESBLs production coexisted with resistance to several other antibiotics because ESBLs are encoded by plasmids, which also carry resistant genes for other antibiotics [33]. It has been found such associated resistance with co-trimoxazole (85.1\%), gentamicin (44.7\%) and fluoroquinolones (84.4\%). In this study aztreonam, ampicillin, amoxyclauve were found $95.0 \%-100 \%$ resistant which is an agreement with other studies [10] [23]. In the present study ceftriaxone, ceftazidime and cefotaxime were found $80.8 \%$, 81.2\% and 77.3\%, which correlates with the study done by Sasirekha et al. [10] and Singh and Goyal [34] which was found $84 \%$ resistance to cefotaxime and $75 \%$ and $85 \%$ resistant for ceftriaxone and ceftazidime respectively [10] [34]. Aminoglycosides have good activity against clinically important gram negative bacilli [35]. In the present study $82.1 \%$ isolates were susceptible to amikacin followed by gentamicin (41.8\%). This is similar to Sasirekha et al. [10]. Several studies showed that amikacin was more sensitive than gentamicin; however, if it is used irrationally, then it may also become resistant. In another study it was reported that gentamicin was 59.0\% resistant in India [10] and 55.5\% in Bangladesh [21]. These variations may be due to increased use of gentamicin, caused by selection pressure of aminoglycosides in different region [36]. Carbapenem is the drug of choice for many infections caused by Gram positive and Gram negative bacteria [23]. In this study imipenem was 100\% sensitive. These findings were similar to study done by Haque and Salam [21]; however, another study showed 3.1\% resistant to imipenem in Bangladesh [21] [37]. Amikacin was the second most common sensitive drug after imipenem. Therefore, these drug resistant bacteria have limited therapeutic options and necessitated the increased use of carbapenem. Beta lactamases are found in K. pneumoniae as K. pneumoniae carbapenemase (KPC) which is resistant to imipenem and has been spread worldwide [38]. Therefore, there is a very limited option to treat imipenem resistant strains; in that situation, colistin may be the drug of choice [39], though it has many side effects. Since co-resistance to non $\beta$ lactam antibiotics like ciprofloxacin, co-trimoxazole and gentamicin was observed, amikacin and nitrofurantoin were found to be alternatives for treating such patients at low cost.

\section{Conclusion}

In conclusion, skin wound is the most commonly infected by ESBL producing E. coli followed by Proteus spe- 
cies with reduced sensitivity profiles among the GNB. An indiscriminate use of the higher antibiotics should be restricted as far as possible. The infection control programs should be monitored continuously in hospital. As a developing country adequate laboratory facilities should be provided to diagnose.

\section{Acknowledgements}

We are grateful to Prof. Nobumichi Kobayashi, Sapporo Medical University of Japan for providing with us Antibiotic disc and Double disc containing 3GCs with Clavulanic acid.

\section{References}

[1] DiNubile, M.J. and Lipsky, B.A. (2004) Complicated Infections of Skin and Skin Structures: When the Infection Is More than Skin Deep. Journal of Antimicrobial Chemotherapy, 53, ii37-ii50. http://dx.doi.org/10.1093/jac/dkh202

[2] Shahcheraghi, F., Nasiri, S. and Noveiri, H. (2009) Detection of Extended-Spectrum Î²-Lactamases (ESBLs). Archives of Clinical Infectious Diseases, 4, 65-70.

[3] Chaudhary, U. and Aggarwal, R. (2004) Extended Spectrum Beta Lactamases (ESBL)—An Emerging Threat to Clinical Therapeutics. Indian Journal of Medical Microbiology, 22, 75-80.

[4] Paterson, D.L. and Bonomo, R.A. (2005) Extended-Spectrum Beta-Lactamases: A Clinical Update. Clinical Microbiology Reviews, 18, 657-686. http://dx.doi.org/10.1128/CMR.18.4.657-686.2005

[5] Perez, F., Endimiani, A., Hujer, K.M. and Bonomo, R.A. (2007) The Continuing Challenge of ESBLs. Current Opinion in Pharmacology, 7, 459-469. http://dx.doi.org/10.1016/j.coph.2007.08.003

[6] Andrews, J. (2012) British Society for Antimicrobial Chemotherapy (BSAC) Detection of Extended-Spectrum $\beta$-Lactamases (ESBLs) in E. coli and Klebsiella Species. http://bsac.org.uk/wp-content/uploads/2012/02/Ecoliklebsiella.pdf

[7] Friedman, C., Callery, S., Jeanes, A., Piaskowski, P. and Scott, L. (2005) Best Infection Control Practices for Patients with Extended Spectrum Beta Lactamase Enterobacteriacae. International Infection Control Council.

[8] Pitout, J.D.D. and Laupland, K.B. (2008) Extended Spectrum $\beta$ Lactamase Producing Enterobacteriaceae: An Emerging Public Health Concern. Lancet Infectious Diseases, 8, 159-166. http://dx.doi.org/10.1016/S1473-3099(08)70041-0

[9] Shivaprakasha, S., Radhakrishnan, K., Gireesh, A.R. and Shamsul Karim, P.M. (2007) Routine Screening for ESBL Production. A Necessity of Today. Internet Journal of Microbiology, 3.

[10] Sasirekha, B., Manasa, R., Ramya, P. and Sneha, R. (2010) Frequency and Antimicrobial Sensitivity Pattern of Extended Spectrum $\beta$-Lactamases Producing Esch. coli and Klebsiella pneumoniae Isolated in a Tertiary Care Hospital. Al Ameen Journal of Medical Science, 3, 265-271.

[11] Shobha, K.L., Gowrish, R.S., Sugandhi, R. and Sreeja, C.K. (2007) Prevalence of Extended Spectrum $\beta$ Lactamases in Urinary Isolates of Escherichia coli, Klebsiella and Citrobacter Species and Their Antimicrobial Susceptibility Pattern in Tertiary Care Hospital. Indian Journal for the Practising Doctor, 3, 1-2.

[12] Agrawal, P., Ghosh, A., Kumar, S., Basu, B. and Kapil, K. (2008) Prevalence of Extended Spectrum $\beta$ Lactamases among E. coli and K. pneumoniae Isolates in Tertiary Care Hospital. Indian Journal of Pathology and Microbiology, 51, 139-142. http://dx.doi.org/10.4103/0377-4929.40428

[13] Yushau, M., Aliyu, H.M., Kumurya, A.S. and Suleiman, K. (2010) Prevalence of Extended Spectrum $\beta$ Lactamases among Enterobacteriaceae in Murtala Mohammed Specialist Hospital, Kano, Nigeria. Bayero Journal of Pure and Applied Sciences, 3, 169-172.

[14] Hawkey, P.M. (2008) Prevalence and Clonality of Extended-Spectrum $\beta$-Lactamases in Asia. Clinical Microbiology and Infection, 14, 159-165. http://dx.doi.org/10.1111/j.1469-0691.2007.01855.x

[15] Rahman, M.M., Haque, J.A., Hossain, M.A., Sultana, R. and Islam, F. (2004) Prevalence of Extended Spectrum Beta Lactamase-Producing Escherichia coli and Klebsiella pneumoniae in an Urban Hospital in Dhaka Bangladesh. International Journal of Antimicrobial Agents, 24, 508-510. http://dx.doi.org/10.1016/j.ijantimicag.2004.05.007

[16] Cheessbrough, M. (2006) District Laboratory Practice in Tropical Countries. Microbiology-2, Cambridge University Press, New York, 184-186.

[17] Clinical Laboratory Standards Institute (2010) Performance Standards for Antimicrobial Disc Susceptibility Testing. 14th Informational Supplement.

[18] Clinical Laboratory Standards Institute (2006) Performance Standards for Antimicrobial Susceptibility Testing. 16th Informational Supplement, M100-S15.

[19] National Committee for Clinical Laboratory Standards (2003) Methods for Dilution Antimicrobial Susceptibility Tests for Bacteria That Grow Aerobically, M7-A6. 
[20] Haque, N., Hossain, M.A., Bilkis, L., Musa, A.K., Mahamud, C., Bari, M.S., et al. (2009) Antibiotic Susceptibility Pattern of Staphylococcus epidermidis. Mymensingh Medical Journal, 18, 142-147.

[21] Haque, R. and Salam, M.A. (2010) Detection of ESBL Producing Nosocomial Gram Negative Bacteria from a Tertiary Care Hospital in Bangladesh. Pakistan Journal of Medical Sciences, 26, 887-891.

[22] Jabeen, K., Zafar, A., Hasan, R., et al. (2005) Frequency and Sensitivity Pattern of Extended Spectrum Beta Lactamase Producing Isolates in a Tertiary Care Hospital Laboratory of Pakistan. Journal of Pakistan Medical Association, 55, 436-439.

[23] Ullah, F., Malik, S.A. and Ahmed, J. (2009) Antimicrobial Susceptibility Pattern and ESBL Prevalence in Klebsiella pneumoniae from Urinary Tract Infections in the North-West of Pakistan. African Journal of Microbiology Research, 3 , 670-680.

[24] Ali, A.M. (2009) Frequency of Extended Spectrum Beta Lactamase (ESBL) Producing Nosocomial Isolates in a Tertiary Care Hospital in Rawalpindi. Journal of the Royal Army Medical Corps, 3, 105-109.

[25] Babypadmini, S. and Appalaraju, B. (2004) Extended Spectrum Beta Lactamases in Urinary Isolates of Escherichia coli and Klebsiella pneumoniae-Prevalence and Susceptibility Pattern in a Tertiary Care Hospital. Indian Journal of Medical Microbiology, 22, 172-174.

[26] Sharma, S., Bhat, G.K. and Shenoy, S. (2007) Virulence Factors and Drug Resistance in Escherichia coli Isolated from Extra Intestinal Infections. Indian Journal of Medical Microbiology, 25, 369-373. http://dx.doi.org/10.4103/0255-0857.37341

[27] Naehi, L., Shahcheraghi, F., Nikbin, V.S. and Nematzadeh, S. (2010) PER, CTX-M, TEM and SHV Beta Lactamases in Clinical Isolates of Klebsiella pneumoniae Isolated from Tehran, Iran. Iranian Journal of Basic Medical Sciences, 13, 111-118.

[28] Lal, P., Kapil, A., Das, B.K. and Sood, S. (2007) Occurrence of TEM and SHV Gene in Extended Spectrum Beta Lactamases (ESBLs) Producing Klebsiella spp. Isolated from a Tertiary Care Hospital. Indian Journal of Medical Research, 125, 173-178.

[29] Sharma, J., Sharma, M. and Roy, P. (2010) Detection of TEM and SHV Genes in Escherichia coli and Klebsiella pneumoniae Isolates in a Tertiary Care Hospital from India. Indian Journal of Medical Microbiology, 132, 332-336.

[30] Tenover, F.C., Mohammed, M.J., Gorton, T.S. and Dembek, Z.F. (1999) Detection and Reporting of Organisms Producing Extended Spectrum Beta-Lactamases: Survey of Laboratories in Connecticut. Journal of Clinical Microbiology, 37, 4065-4070.

[31] Paterson, D.L. and Yu, V.L. (1999) Extended Spectrum Beta Lactamases: A Call for Improved Detection and Control. Clinical Infectious Diseases, 29, 1419-1422. http://dx.doi.org/10.1086/313559

[32] Asna, S.M.Z. and Haq, J.A. (2000) Decrease of Antibiotic Resistance in Salmonella typhi Isolated from Patients Attending Hospitals of Dhaka City over a 3 Year Period. International Journal of Antimicrobial Agents, 16, 249-251. http://dx.doi.org/10.1016/S0924-8579(00)00230-2

[33] Paterson, D.L., Ko, W.C., Gottberg, V., Mohapatra, S., Casellas, J.M., Goossens, H., et al. (2004) Antibiotic Therapy for Klebsiella pneumoniae Bacteriamia: Implications of Production of Extended-Spectrum $\beta$-Lactamases. Antibiotics for ESBL Producers CID, 39, 31-37.

[34] Singh, N.P. and Goyal, R. (2003) Changing Trends in Bacteriology of Burns in the Burns Unit, Delhi, India. Burns, 29, 129-132. http://dx.doi.org/10.1016/S0305-4179(02)00249-8

[35] Gonzalez, L.S. and Spencer, J.P. (1998) Aminoglycosides: A Practical Review. American Family Physician, 58, 18111820.

[36] Miller, G.H. and Sabatelli, F.J. (1997) The Most Frequent Aminoglycoside Resistance Mechanisms—Changes with Time and Geographic Area: Areflection of Aminoglycoside Usage Patterns? Aminoglycoside Resistance Study Groups. Clinical Infectious Diseases, 24, 46-62. http://dx.doi.org/10.1093/clinids/24.Supplement_1.S46

[37] Rashid, A., Chowdhury, A., Rahman, S.H., Begum, S.A. and Muazzam, N. (2007) Infections by Pseudomonas aeruginosa and Antibiotic Resistance Pattern of the Isolates from Dhaka Medical College Hospital. Bangladesh Journal of Medical Microbiology, 1, 48-51.

[38] Rhee, Y.J., Park, Y.K., Lee, M.Y., Peck, K.R. and Soo, K.K. (2010) KPC-Producing Extreme Drug-Resistant Klebsiella pneumoniae Isolated from a Patient with Diabetes Mallitus and Chronic Renal Failure on Haemodialysis in South Korea. Antimicrobial Agents and Chemotherapy, 54, 2278-2279. http://dx.doi.org/10.1128/AAC.00011-10

[39] Amaya, E., Caceres, M., Fang, H., Ramirez, A.T., Palmgren, A.C., Nord, C.E. and Weintraub, A. (2009) Extended Spectrum Beta Lactamase-Producing Klebsiella pneumoniae in a Neonatal Intensive Care Unit in Leon, Nicaragua. International Journal of Antimicrobial Agents, 33, 386-387. http://dx.doi.org/10.1016/j.ijantimicag.2008.10.004 